\title{
Research on Clothing Pressure Measuring Equipment Based on LabVIEW
}

\author{
Jiawei Shao ${ }^{\mathrm{a}}$, Zhi Gao ${ }^{\mathrm{b}}$ \\ School of Mechanical \& Power Engineer, East China University of Science and Technology, \\ Shanghai 200237, China \\ a13382483279@163.com, bgaozhi@ecust.edu.cn
}

\begin{abstract}
Keywords: Clothing Pressure, Tension Sensor, Displacement Sensor, Worm Type of Reduction Gearing, Data Acquisition.
\end{abstract}

\begin{abstract}
In recent years, pressure clothing has become a research hotspot at home and abroad in order to satisfy the disease treatment, body care, kinematics function requirements. In order to accurately measure the size of the clothing pressure, this paper proposes a new kind of clothing pressure measuring equipment. The mechanical structure is constituted by the tension sensor, the displacement sensor and worm type of reduction gearing. Then we design the circuit to control the data acquisition. Meanwhile, we develop the human-computer interaction system based on LabVIEW to implement the visualization and storage of experimental data. The whole equipment has reasonable structure, good stability, precision and sensitivity to satisfy the use requirement.
\end{abstract}

\section{Introduction}

Pressure garments are well known in our daily life, such as varicose veins socks, antithrombotic belt and all kinds of pressure of corsets, pants, etc. These garments promote blood circulation, relieve or improve the pressure of limb vein and vein valve effectively to prevent blood clots travel by pressure [1]. However, the pressure is not the bigger the better, or not the smaller the better. So it's important to measure the pressure of clothing precisely.

So far, all the devices are committed to direct measurement on the domestic market, but this kind of measurement method is limited to the particularity of surface pressure measurement of the human body. This kind of measurement method is limited to the flexible sensor measurement [2]. Currently, there are two main types of sensors. The one is a flexible thin-film pressure sensor based on the piezoelectric principle, which is represented by the TEKSCAN pressure distribution test system in the United States [3]. The other is based on airbag type pressure sensor, which is represented by Japan.

However, the principle and use of these sensors shows that both types of sensors are clearly deficient. Piezoelectric flexible thin-film sensor has large measurement errors, reproducibility difference and slow dynamic response [4]. The airbag type pressure sensor has large measurement errors and slow response due to the compressibility of the gas [5]. These factors affect the accuracy of measurement.

In order to avoid the disadvantages of current clothing pressure measuring equipment, this paper puts forward a new type of clothing pressure measurement equipment and develops a corresponding human-computer interaction system to complete the equivalent indirect measurement and ensure the accuracy of clothing pressure.

\section{Design of mechanical structure of clothing pressure measuring equipment}

\subsection{Machinery construction}

The technical solution of mechanical structure of the equipment: Using the measuring range $0-10 \mathrm{~kg}$ pull sensor and weighing amplifier as the measuring element. Using the displacement sensor with $250 \mathrm{~mm}$ effective stroke and the $0.08 \%$ independent linear precision as the finalizing element of the equivalent arm shape. The adjustment of various sizes of the equivalent arm pressure suit model is achieved through the mechanical structure of the worm reducer, the strong string, the guide rail and 
the sliding block connection. Figure 1 shows the model main view of the clothing pressure measurement device and a physical map of the clothing pressure measurement equipment.
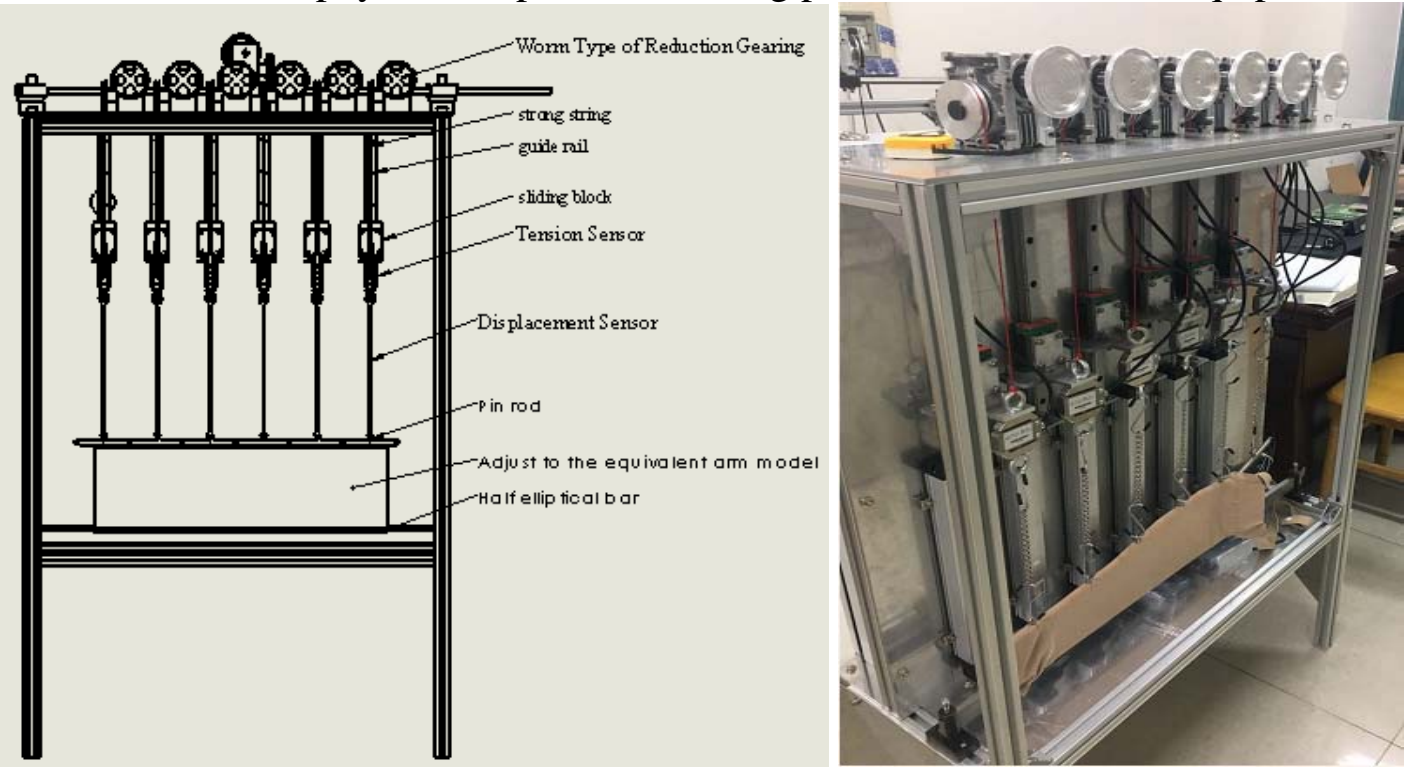

Fig. 1 The Clothing Pressure Measuring Equipment

\subsection{Machinery control sequence}

The order of mechanical control of the clothing pressure measuring equipment: By turning the hand wheel of worm gear and worm gear reducer, pull the strong cord winding on the reducer to promote the slider sliding on the guide rail. Then it drives the tension sensor and pulls the scale of displacement sensor. At last, pull pin bar to adjust to the equivalent model of the arm. Figure 2 is the mechanical control sequence diagram.

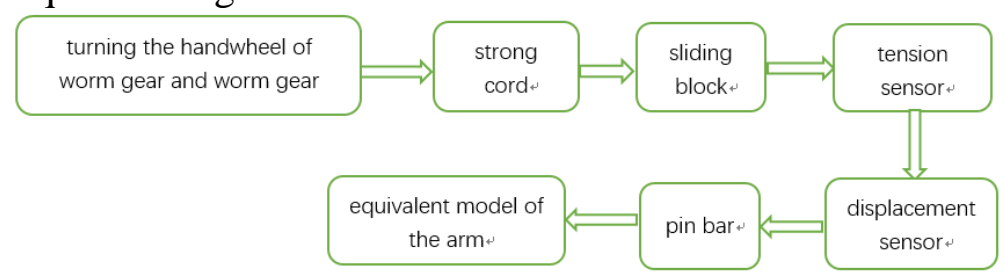

Fig. 2 Mechanical Control Sequence Diagram

\subsection{Transformation theory of equivalent arm model}

The equipment of this research is mainly applied to the pressure measurement of the cylinder pressure clothing, such as the arm pressure suit. When we do the experiment, one end of the arm pressure suit is put on the pin and set with the ring screw. The other end was fixed on the bottom half ellipse bar. The distance $\mathrm{H}$ between the top pin and the lower end of the oval bar can be converted into the diameter of the pressure suit. Figure 3 shows the position of displacement sensor when the pin is in contact with the semi-ellipse bar. Figure 4 shows a diagram of the pin pulled up to $\mathrm{H}$ height. The size of $\mathrm{H}$ is reflected in real-time by the displacement sensor.

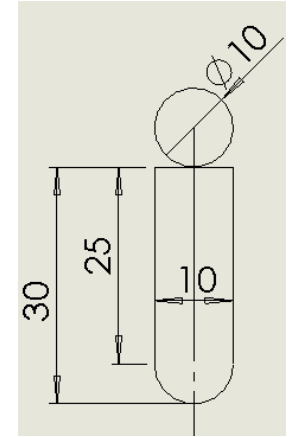

Fig. 3 Displacement Sensor Zero Setting

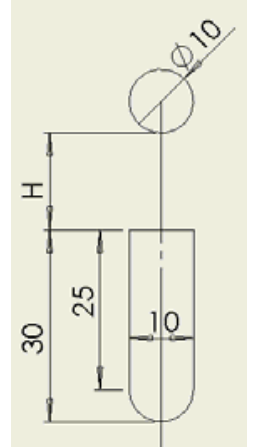

Fig.4 Displacement Sensor H Setting

According to Figure 4, the perimeter of the arm pressure suit under two bars is available.

$L=91.4+2 H$ 
The diameter $\mathrm{D}$ of equivalent arm pressure is converted.

$$
D=L / \pi=(91.4+2 H) / \pi
$$

So you can determine the diameter of the arm pressure suit by adjusting the height $\mathrm{H}$ of the displacement sensor. A sample of any size can be measured to make the measurement of the device universal . At the same time, the device's equivalent indirect measurement avoids the limitation of direct measurement of human surface.

\section{Design of data collection circuit of clothing pressure measuring equipment}

The basic task of the data collection circuit is to automatically gather information from a sensor or other measuring device in the form of simulate quantity or digital quantity [6]. It requires hardware structures, such as computers, data collection cards, signal conditioning circuits and sensors. In this data acquisition circuit, we adopt NI USB - 6210 data acquisition card. It can be directly connected to the computer via USB line, plug and play, easy to use.

This design principle of data collection circuit: Magnifying the signal from the pull sensor by pulling the weight amplifier. Then the data collection card will be connected to the computer via the USB cable, and the data will eventually be imported into the computer. Figure 5 shows the data collection circuit layout and the object map.

The two figures below show that the $1 \# \sim 7$ \# pull weight amplifier are assigned at the bottom. At the middle, the NI USB-6210 data collection card is assigned on the left. The ground copper and $24 \mathrm{~V}$ switch power supply are assigned on the right. At the top, the circuit breaker, fuse and terminals are placed from left to right to ensure the safety of the circuit.

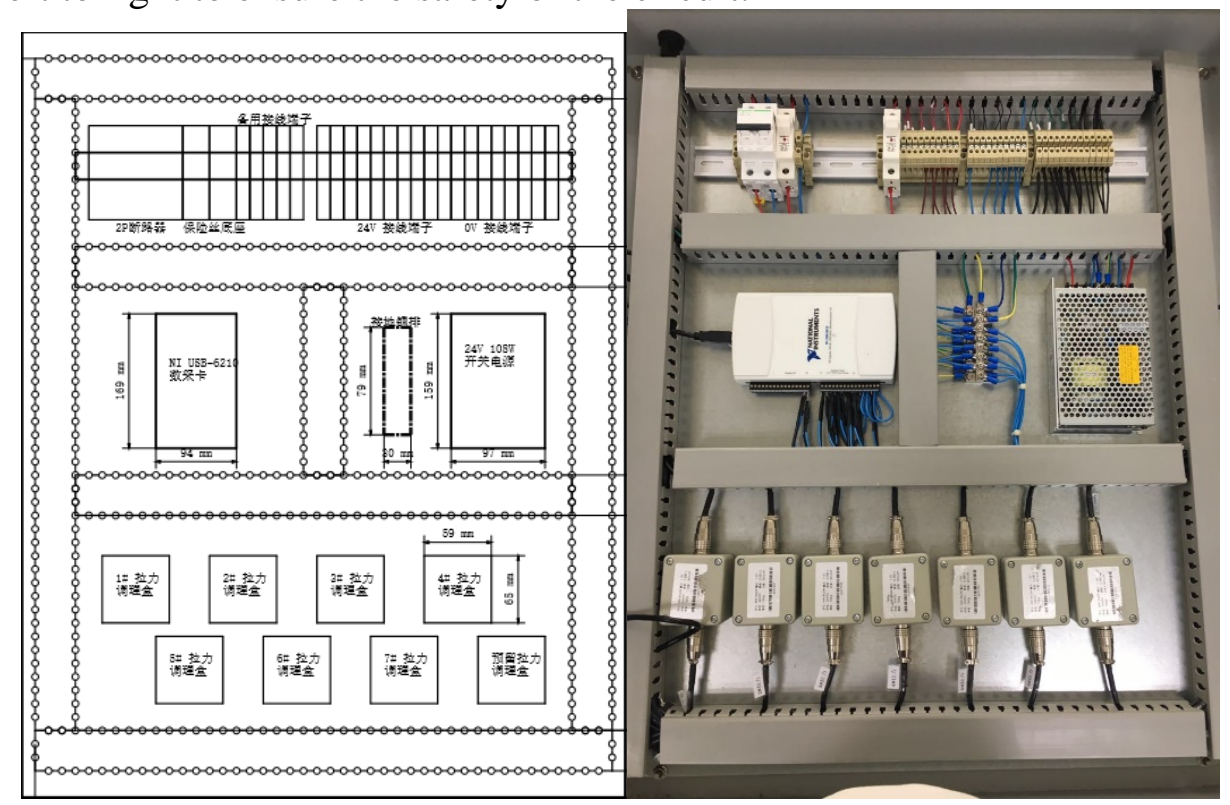

Fig. 5 Data Acquisition Circuit

\section{The development of the interactive system of clothing pressure measurement equipment}

The human-computer interaction system of the measuring equipment is a set of data acquisition system based on LabVIEW, which mainly includes sensor measurement calibration, data real-time display and data storage.

\subsection{Calibration of sensors}

This equipment adopts the pressure sensor with measuring range of $0 \sim 10 \mathrm{KPa}$ and the output voltage of $1 \sim 5 \mathrm{v}$. Then we complete calibration through the weight of $1 \sim 10 \mathrm{~kg}$. We obtain the linear relation curve between the input pressure and the output voltage. For example, we hang $7 \mathrm{~kg}$ weight. Then we input the target pressure value $68.6 \mathrm{~N}$, the measured voltage value shows $3.814 \mathrm{~V}$. After 
waiting for voltage stability, it can be thought that the voltage value corresponds to the target load value.

Two or more points can be calibrated when the tension sensor is calibrated. After the calibration, the calibration curve is displayed in the top right of the chart. The calibration results will be displayed in the form of $y=k x+b$. The curve is the tension sensor calibration curve. The front panel and program block diagram of the calibration function is shown in figure 6 .
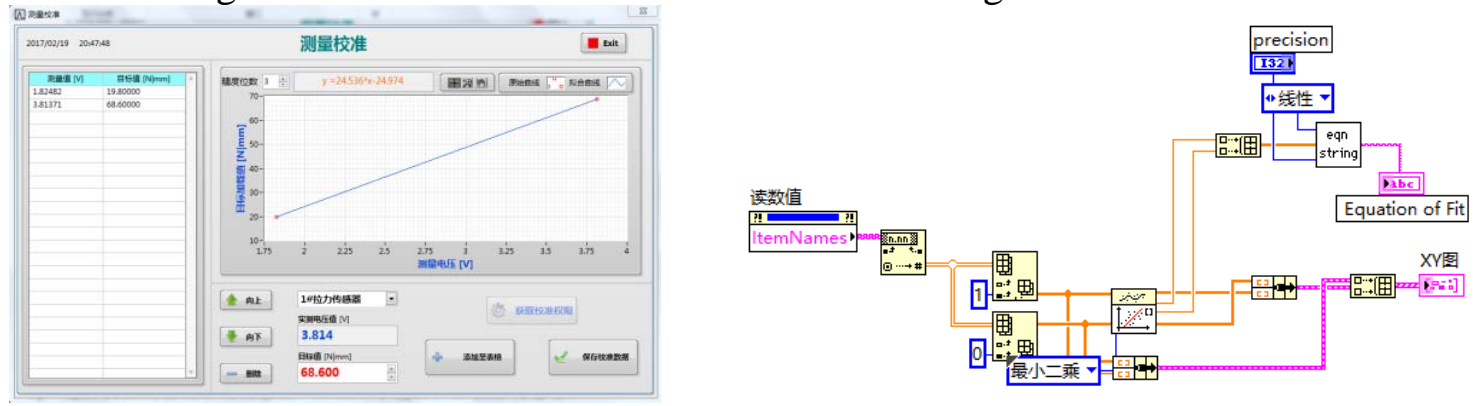

Fig. 6 Calibration

\subsection{Data measurement display and storage}

The measurement part of arm pressure suit completes the real-time data display of displacement sensor and strain sensor, zero operation, single point measurement and composite measurement and table data display. As shown in figure 7, the left-hand side of the software interface is the real-time data display area. When the program runs, it shows the displacement values and the pull values of the six locations. In the upper right part of the interface, the system switches the single point measurement and composite measuring. The right of figure 7 is a program diagram for measuring functionality. Click the add data button to add the data to the table in the lower right part, as shown in figure 8 . The other of figure 8 shows the program diagram for the data display. Click save the table data button to save the table data to the Excel table, as shown in figure 9. The other of figure 9 is a program block diagram for data storage.

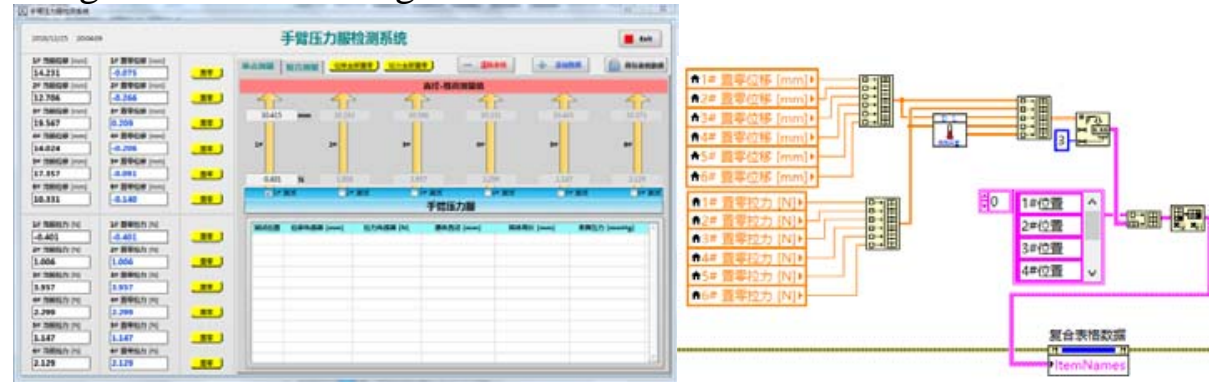

Fig. 7 Data Measurement

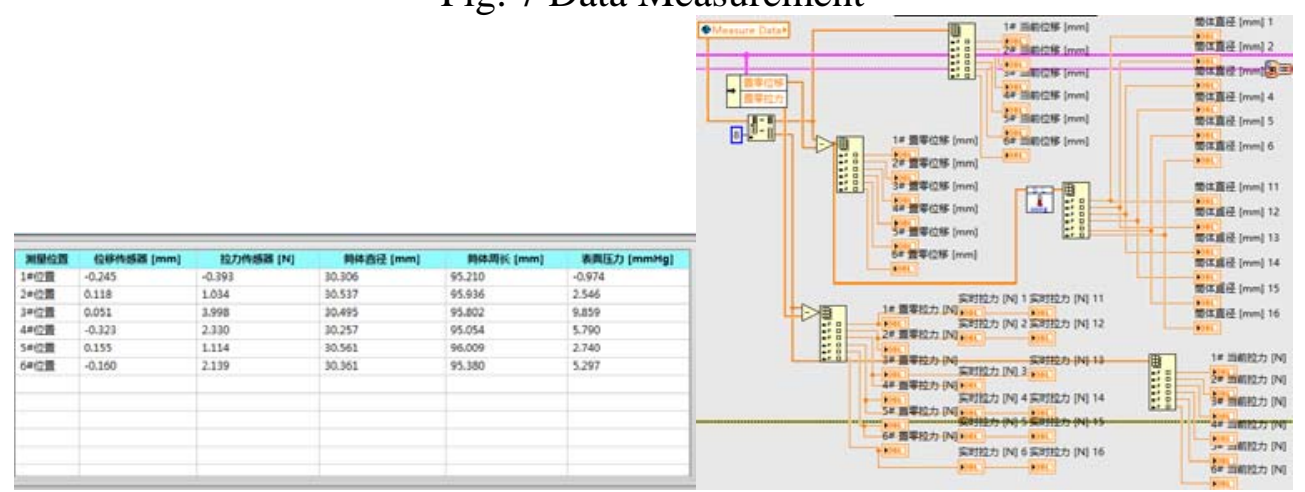

Fig. 8 Data Display 


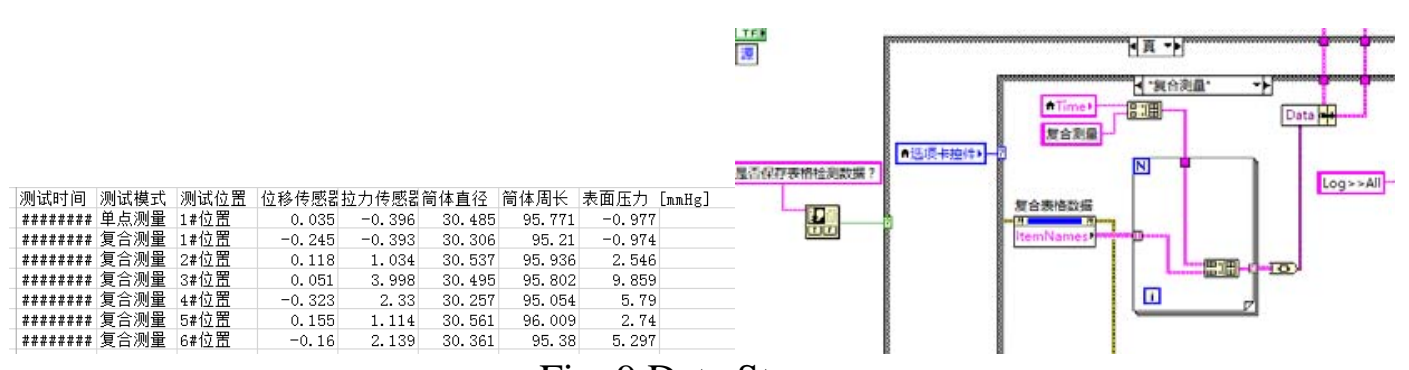

Other panels are not detailed.

Fig. 9 Data Storage

\section{Conclusion}

By comparing the measured results of the pressure garments with their product parameters, the experimental results show that the design of the pressure measurement system is feasible. It can adjust to any size and check the clothing pressure of different dimensional state. It shows that the equipment is widely used and versatile.

Use standard load weights for calibration. The equation between the input pressure and the output voltage combined with the least square method shows strong linear correlation and good test performance. The device can measure multiple parts of clothing pressure and can record data in real time. It provides the basic data for the design of the clothing pressure comfort index and the clothing pressure data analysis. In this way, we can change the status quo of traditional clothing.

With the progress of sensor technology and based on the development of computer bus technology, instrument control and data acquisition technology, the clothing pressure measurement system provides technical support and equipment support. At the same time, with the further development of computer technology and sensing technology, the clothing pressure measurement system will be improved.

\section{References}

[1] Xu Xian-lin. Study of Surgical Pressure Bandage [D]. Degree Thesis of Tianjin University of Technology. 2002: 1-2

[2] Yi Xu. Flexible shear-stress sensor skin and its application to unmanned aerial vehicles [J]. Sensors and Actuators A: Physical, 2003, 105(3): 321-329.

[3] Wang Yu-Qiao, Li Wei, Song Xing-Yuan. The Pressure Distribution Measurement System Based on LabVIEW and TekSCan [J] Instrumentation Customer. 2004, 11(1): 52-57.

[4] FISHER G. Intelligent textiles for medical and monitoring applications [J]. Technical Textiles International, 2001, 10(6): 11-14.

[5] Song Xiao-xia. The Relationship between The Garment Pressure of Knitting Underwear and The Comfort of Human Body [J]. Knitting Industries. 2007, 4: 33-37.

[6] Liu Jun-hua. Virtual Instrument Design Based on LabVIEW [M]. Beijing Electronic Industry Press, 2003. 3-10. 